\title{
Sobolev homeomorphisms are dense in volume preserving automorphisms
}

\author{
Assis Azevedo ${ }^{\mathrm{a}, *}$, Davide Azevedo ${ }^{\mathrm{b}}$, Mário Bessac ${ }^{\mathrm{c}}$, Maria Joana Torres ${ }^{\mathrm{a}}$ \\ ${ }^{a}$ CMAT e Departamento de Matemática, Universidade do Minho, \\ Campus de Gualtar, 4700-057 Braga, Portugal \\ ${ }^{b}$ Departamento de Matemática, Universidade Federal da Bahia, \\ Av. Ademar de Barros s/n, 40170-110 Salvador, Brasil \\ ${ }^{c}$ Departamento de Matemática, Universidade da Beira Interior, \\ Rua Marquês d'Ávila e Bolama, 6201-001 Covilhã, Portugal.
}

\begin{abstract}
In this paper we prove a weak version of Lusin's theorem for the space of Sobolev- $(1, p)$ volume preserving homeomorphisms on closed and connected $n$-dimensional manifolds, $n \geq 3$, for $p<n-1$. We also prove that if $p>n$ this result is not true. More precisely, we obtain the density of Sobolev- $(1, p)$ homeomorphisms in the space of volume preserving automorphisms, for the weak topology. Furthermore, the regularization of an automorphism in a uniform ball centered at the identity can be done in a Sobolev- $(1, p)$ ball with the same radius centered at the identity.
\end{abstract}

Keywords: Lusin theorem, volume preserving, Sobolev homeomorphism

\section{Introduction}

J. E. Littlewood formulated the classic Lusin theorem by saying that 'every measurable function is nearly continuous'. In the spirit of this formulation, we prove that 'every measurable volume preserving map is nearly a Sobolev-(1,p) volume preserving homeomorphism'. More precisely, we prove a weak version of Lusin's theorem (see Theorem A in Section 4) for the

${ }^{*}$ Corresponding author

Email addresses: assis@math.uminho.pt (Assis Azevedo), davidemsa@gmail.com (Davide Azevedo), bessa@ubi.pt (Mário Bessa), jtorres@math.uminho.pt (Maria Joana Torres) 
space of Sobolev- $(1, p)$ volume preserving homeomorphisms on closed and connected $n$-dimensional manifolds, $n \geq 3$, for $p<n-1$.

This theorem generalizes for the Sobolev setting previous continuous versions proved by Oxtoby [10], White [12], Alpern [1] and Alpern and Edwards [2].

The proof of Theorem A is based on a key perturbation result (Theorem 3.1). This perturbation theorem is the Sobolev version of a classical result proved by Oxtoby and Ulam [9] which, in rough terms, says that given any $\varepsilon>0$ and any two sets of distinct $N$ points $\left\{P_{i}\right\}_{i=1}^{N}$ and $\left\{Q_{i}\right\}_{i=1}^{N}$ in $\mathbb{R}^{n}$ such that $P_{i}$ is $\varepsilon$-close to $Q_{i}$ for all $i$ we can construct a volume preserving homeomorphism $h \varepsilon$-close to the identity which maps some neighbourhood of $P_{i}$ by simple translation onto a neighbourhood of $Q_{i}$. An explicit construction of $h$ can be seen in $[2,3]$. Yet, the strategy used in $[2,3]$ cannot be applied in our Sobolev setting because we need to control the $L^{p}$-norm of the partial derivatives of $h$. To obtain this control we define $h$ as the composition of $2 N$ simpler perturbations, which locally is a composition of just two perturbations.

Besides its intrinsic importance in Analysis, the weak Lusin theorem in the volume preserving class was crucial in a proof given in [3] of the result of Oxtoby and Ulam, that ergodicity is generic for measure preserving homeomorphisms of compact manifolds. Therefore, a Sobolev version of this theorem could be useful in the study of dynamical properties in the Sobolev class. Indeed, homeomorphisms on the Sobolev class gain significance presently in applications to certain type of PDE's in nonlinear elasticity (see the BallEvans Problem in [7]), in ergodic theory (genericity of infinite topological entropy in $[5,4]$ and also subjects correlated with the closing lemma (see again [5]). We believe that building bridges connecting these two areas could be of utmost interest both in applications and fundamental mathematics.

This paper is organized as follows. In Section 2 we introduce the space of automorphisms and the space of Sobolev- $(1, p)$ volume preserving homeomorphisms. In Section 3 we prove the perturbation result (Theorem 3.1). Finally, in Section 4, we prove a weak version of Lusin's theorem (Theorem A) for the space of Sobolev- $(1, p)$ volume preserving homeomorphisms, for $p<n-1$ and we present a counterexample (see Example 4.1) for $p>n$. 


\section{Preliminaries}

Throughout the article $X$ is a smooth closed connected Riemannian manifold of dimension $n$ and $d$ is the geodesic distance on $X$. We denote the Euclidean norm in $\mathbb{R}^{n}$ by $|\cdot|$. We shall denote by $\lambda$ the volume measure on both $X$ and $\mathbb{R}^{n}$.

\subsection{Automorphisms and homeomorphisms of $(X, \lambda)$}

An automorphism of the underlying Borel measure space $(X, \lambda)$ is a bijection $g: X \rightarrow X$ such that both $g$ and $g^{-1}$ are measurable functions and $\lambda(B)=\lambda(g(B))=\lambda\left(g^{-1}(B)\right)$ for all measurable sets $B$. Automorphisms which differ on a set of measure zero will be identified. We denote by $\mathcal{G}(X)$ the space of automorphisms of $(X, \lambda)$. We shall consider two topologies on $\mathcal{G}(X)$ : the weak topology given by the metric $\rho(f, g)=\inf \{\delta: \lambda\{x:$ $d(f(x), g(x)) \geq \delta\}<\delta\}$, and the uniform topology defined by the metric $\|f-g\|_{\infty} \equiv \sup \operatorname{ess}_{x \in X} d(f(x), g(x))$. The space $\mathcal{G}(X)$ is topologically complete with the weak topology (see [6]) and complete with the uniform topology. Thus, with each of these topologies, $\mathcal{G}(X)$ is a Baire space. We denote by $\mathcal{M}(X)$ the subspace of all homeomorphisms in $\mathcal{G}(X)$, endowed with the uniform topology. This space is topologically complete (see [11]). We shall call volume preserving homeomorphisms of $X$ the elements in $\mathcal{M}(X)$.

\subsection{Sobolev maps}

Let $\Omega$ be an open bounded subset of $\mathbb{R}^{n}$ with Lipschitz boundary and let $1 \leq p \leq \infty$. Given a set $A \subseteq \mathbb{R}^{n}$ and $\delta>0$ we denote by $V_{\delta}(A)$ the set $\left\{x \in \mathbb{R}^{n}: \inf _{a \in A}|x-a|<\delta\right\}$.

Recall that a measurable map $f=\left(f_{1}, \ldots, f_{n}\right): \Omega \rightarrow \mathbb{R}^{n}$ is in the Sobolev class $W^{1, p}\left(\Omega, \mathbb{R}^{n}\right)$ if, for all $i=1, \ldots, n, f_{i}$ and all its distributional partial derivatives $\partial f_{i} / \partial x_{j}$ are in $L^{p}(\Omega)$.

We endow $W^{1, p}\left(\Omega, \mathbb{R}^{n}\right)$ with the norm defined by

$$
\|f\|_{1, p}=\|f\|_{p}+\|D f\|_{p}, \quad \forall f \in W^{1, p}\left(\Omega, \mathbb{R}^{n}\right),
$$

where $\|f\|_{p}=\max _{i}\left\|f_{i}\right\|_{p}$ and $\|D f\|_{p}=\max _{i, j}\left\|\frac{\partial f_{i}}{\partial x_{j}}\right\|_{p}$.

We shall be interested only on Sobolev maps that are continuous up to the boundary. More precisely, we will consider the space

$$
W^{1, p}\left(\Omega, \mathbb{R}^{n}\right) \cap C^{0}\left(\bar{\Omega}, \mathbb{R}^{n}\right) .
$$


The (natural) norm in this space is equivalent to the one defined by

$$
\|f\|_{\infty}+\|D f\|_{p}
$$

since $C^{0}(\bar{\Omega}, \mathbb{R})$ is compactly included in $L^{p}(\Omega)$.

Remark 2.1. If $p>n$ then $W^{1, p}\left(\Omega, \mathbb{R}^{n}\right) \subseteq C^{0}\left(\bar{\Omega}, \mathbb{R}^{n}\right)$ and the norms on $W^{1, p}\left(\Omega, \mathbb{R}^{n}\right)$ defined above are equivalent.

Finally we define the Sobolev space we are going to work with.

Definition 2.1. We define $\mathbb{W}_{\lambda}^{1, p}(\Omega)$ as the set of all volume preserving homeomorphisms $f: \Omega \rightarrow \Omega$ such that $f \in W^{1, p}\left(\Omega, \mathbb{R}^{n}\right) \cap C^{0}\left(\bar{\Omega}, \mathbb{R}^{n}\right)$. In this space we consider the natural metric defined by

$$
d_{\mathbb{W}_{\lambda}^{1, p}(\Omega)}(f, g)=\|f-g\|_{\infty}+\|D(f-g)\|_{p},
$$

for $f, g \in \mathbb{W}_{\lambda}^{1, p}(\Omega)$. We shall call Sobolev- $(1, p)$ volume preserving homeomorphisms of $\Omega$ the elements in $\mathbb{W}_{\lambda}^{1, p}(\Omega)$.

For simplicity we will denote $d_{\mathbb{W}_{\lambda}^{1, p}(\Omega)}(f, g)$ by $\|f-g\|_{\infty ; 1, p}$.

Since $\mathcal{M}_{\lambda}(\bar{\Omega})$ is topologically complete and $W^{1, p}\left(\Omega, \mathbb{R}^{n}\right) \cap C^{0}\left(\bar{\Omega}, \mathbb{R}^{n}\right)$ is complete, we have the following.

Proposition 2.1. $\mathbb{W}_{\lambda}^{1, p}(\Omega)$ is a Baire space.

Finally, we define a similar space for the manifold $X$. We denote by $\mathbb{W}_{\lambda}^{1, p}(X)$ the space of volume preserving homeomorphisms on $X$ which in all local charts are Sobolev- $(1, p)$ maps.

\section{A key perturbation theorem: ellipsoids}

In this section we prove a key perturbation result which is the main ingredient to prove a volume preserving Sobolev weak Lusin theorem. Let $I^{n}=[0,1]^{n}$ stand for the $n$-dimensional unit cube. 
Theorem 3.1. Let $n \geq 3, \varepsilon>0$ and $N \in \mathbb{N}$. Let $\left\{P_{i}\right\}_{i=1}^{N}$ and $\left\{Q_{i}\right\}_{i=1}^{N}$ be two sets of $N$ distinct interior points of $I^{n}$ such that $\left|P_{i}-Q_{i}\right|<\varepsilon$, for all $i$.

If $p<n-1$, then, for all $k \in \mathbb{N}$ there is $F_{k} \in \mathbb{W}_{\lambda}^{1, p}\left(I^{n}\right)$ such that, for all $i, F_{k}\left(P_{i}\right)=Q_{i}, F_{k}$ sends a neighbourhood of $P_{i}$ by translation onto a neighbourhood of $Q_{i}$, and

$$
\sup _{k}\left\|F_{k}-I d\right\|_{\infty}<\varepsilon, \quad \lim _{k \rightarrow \infty}\left\|F_{k}-I d\right\|_{1, p}=0 .
$$

As a consequence, for $k$ large enough, $\left\|F_{k}-I d\right\|_{\infty ; 1, p}<\varepsilon$.

In addition, the functions $F_{k}$ are $\mathbb{C}^{\infty}$ diffeomorphisms and equal to the identity in a neighbourhood of the boundary of $I^{n}$.

Remark 3.1. Notice that, if $p>n$, the conclusion of this theorem is false unless $P_{i}=Q_{i}$, for all $i$. In fact, taking in mind Remark 2.1, if $\lim _{k \rightarrow \infty}\left\|F_{k}-I d\right\|_{1, p}=$ 0 then also $\lim _{k \rightarrow \infty}\left\|F_{k}-I d\right\|_{\infty}=0$. Hence, $\max _{i=1, \ldots, N}\left|Q_{i}-P_{i}\right|=0$.

The rest of this section is devoted to the proof of Theorem 3.1.

Let $a, b, \mu>0$ with $a \geq b$ and $0<\mu \leq 1$ and consider the ellipsoids

$$
\Sigma_{a, b}=\left\{x \in \mathbb{R}^{n}:\left(\frac{x_{1}}{a}\right)^{2}+\sum_{i=2}^{n}\left(\frac{x_{i}}{b}\right)^{2} \leq 1\right\}, \quad \Sigma_{a, b, \mu}=\Sigma_{(1-\mu) a,(1-\mu) b} .
$$

Notice that $\lambda\left(\Sigma_{a, b}\right)=w_{n} a b^{n-1}$, where $w_{n}$ is the volume of the unitary sphere in $\mathbb{R}^{n}$, and $\mu \lambda\left(\Sigma_{a, b}\right) \leq \lambda\left(\Sigma_{a, b} \backslash \Sigma_{a, b, \mu}\right) \leq n \mu \lambda\left(\Sigma_{a, b}\right)$.

For each $a, b, \mu$, we will define a volume preserving $\mathbb{C}^{\infty}$ diffeomorphism of $\mathbb{R}^{n}, F_{a, b, \mu}$, that is rigid in $\Sigma_{a, b, \mu}$ and is equal to the identity outside $\Sigma_{a, b}$. In order to do that, we first consider a function $h_{\mu} \in C^{\infty}(\mathbb{R})$, strictly decreasing in $] 1-\mu, 1[$ and constant in $]-\infty, 1-\mu]$ and in $\left[1,+\infty\left[\right.\right.$. We let $h_{\mu}$ be defined

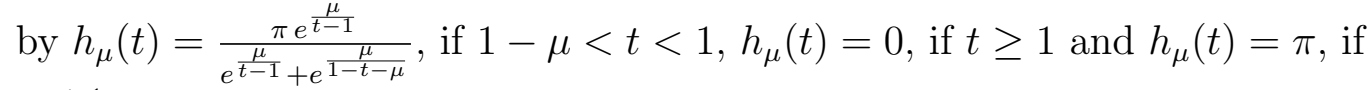
$t \leq 1-\mu$.

Lemma 3.2. In the above conditions, $h_{\mu} \in C^{\infty}(\mathbb{R})$ and $\left|h_{\mu}^{\prime}(t)\right| \leq \frac{2 \pi}{\mu}$, for all $t \in \mathbb{R}$. 
Proof. Since $h_{1}\left(\frac{1}{\mu}(t-(1-\mu))\right)=h_{\mu}(t), t \in \mathbb{R}$, it is enough to show the result for $\mu=1$. But

$$
\begin{aligned}
\frac{1}{\pi}\left|h_{1}^{\prime}(t)\right| & =\frac{e^{-\frac{1}{t}} \cdot e^{\frac{1}{t-1}}}{\left(e^{-\frac{1}{t}}+e^{\frac{1}{t-1}}\right)^{2}}\left(\frac{1}{t^{2}}+\frac{1}{(t-1)^{2}}\right) \\
& =\underbrace{\frac{e^{-\frac{1}{t}}}{t^{2}}}_{\leq 4 e^{-2}}\left(\frac{e^{\frac{1}{t-1}}}{\left(e^{-\frac{1}{t}}+e^{\frac{1}{t-1}}\right)^{2}}\right)+\underbrace{\frac{e^{\frac{1}{t-1}}}{t^{2}}}_{\leq 4 e^{-2}}\left(\frac{e^{-\frac{1}{t}}}{\left(e^{-\frac{1}{t}}+e^{\frac{1}{t-1}}\right)^{2}}\right) \\
& \leq \frac{4 e^{-2}}{e^{-\frac{1}{t}}+e^{\frac{1}{t-1}}}
\end{aligned}
$$

and the conclusion follows, since $e^{-\frac{1}{t}}+e^{\frac{1}{t-1}} \geq 2 e^{-\frac{1}{2 t}+\frac{1}{2(t-1)}} \geq 2 e^{-2}$, remembering that the geometric mean of two numbers is less than or equal to their arithmetic mean.

Consider now the function $F_{a, b, \mu}: \mathbb{R}^{n} \rightarrow \mathbb{R}^{n}$ defined by

$$
F_{a, b, \mu}(x)=\left(x_{1} \cos (\alpha(x))-\frac{a}{b} x_{2} \sin (\alpha(x)), \frac{b}{a} x_{1} \sin (\alpha(x))+x_{2} \cos (\alpha(x)), \bar{x}\right)
$$

where $\bar{x}=\left(x_{3}, \ldots, x_{n}\right)$ and $\alpha: \mathbb{R}^{n} \longrightarrow \mathbb{R}$.

$$
x \mapsto h_{\mu}\left(\sqrt{\left(\frac{x_{1}}{a}\right)^{2}+\sum_{i=2}^{n}\left(\frac{x_{i}}{b}\right)^{2}}\right)
$$

To be precise, we should write $\alpha_{a, b, \mu}$, but we choose to drop the subscripts as it will be clear in the context.

Note that $\left|\frac{\partial \alpha}{\partial x_{1}}\right| \leq \frac{2 \pi}{\mu a}$ and $\left|\frac{\partial \alpha}{\partial x_{i}}\right| \leq \frac{2 \pi}{\mu b}$, if $i \geq 2$.

Lemma 3.3. In the above conditions, $F_{a, b, \mu}$ is a volume preserving $\mathbb{C}^{\infty}$ diffeomorphism, is equal to the identity in $\mathbb{R}^{n} \backslash \Sigma_{a, b}$ and $F_{a, b, \mu}\left(x_{1}, x_{2}, \ldots, x_{n}\right)=$ $\left(-x_{1},-x_{2}, x_{3}, \ldots, x_{n}\right)$ in $\Sigma_{a, b, \mu}$.

In addition, denoting $F_{a, b, \mu}$ by $\left(F_{1}, \ldots, F_{n}\right)$, we have

$$
\forall x \in \mathbb{R}^{n} \quad\left|\frac{\partial F_{i}}{\partial x_{j}}(x)\right| \leq \frac{a}{b} \cdot \frac{6 \pi}{\mu} .
$$

Proof. If $x \in \Sigma_{a, b}$ then $\alpha(x)=\alpha\left(F_{a, b, \mu}(x)\right)$ since

$$
\frac{\left(x_{1} \cos (\alpha(x))-\frac{a}{b} x_{2} \sin (\alpha(x))\right)^{2}}{a^{2}}+\frac{\left(\frac{b}{a} x_{1} \sin (\alpha(x))+x_{2} \cos (\alpha(x))\right)^{2}}{b^{2}}=\frac{x_{1}^{2}}{a^{2}}+\frac{x_{2}^{2}}{b^{2}} \text {. }
$$


Therefore, $F_{a, b, \mu}^{-1}$ is obtained by replacing $\alpha(x)$ by $-\alpha(x)$ in $F_{a, b, \mu}$.

The function $F_{a, b, \mu}$ preserves the volume because the determinant of its jacobian matrix is equal to the determinant of the jacobian matrix of $\left(F_{1}, F_{2}\right)$, which can easily be seen to be equal to 1 . Indeed, for this calculation, we do not need to use the expression of $\alpha$ but only that it is a function of $\left(\frac{x_{1}}{a}\right)^{2}+\sum_{i=2}^{n}\left(\frac{x_{i}}{b}\right)^{2}$.

The conditions on the partial derivatives of $F_{a, b, \mu}$ are simply a consequence of the hypotheses on $a, b, \mu$ and of the inequalities $|\cos (\alpha(x))|,|\sin (\alpha(x))| \leq$ $1,\left|x_{1}\right| \leq a,\left|x_{i}\right| \leq b$, for $i \geq 2$.

A similar result can be obtained for ellipsoids in general position in the space.

Corollary 3.4. Let $a, b, \mu \in \mathbb{R}$, with $a \geq b>0,0<\mu<1$. If $F_{a, b, \mu, T}=$ $T \circ F_{a, b, \mu} \circ T^{-1}$, where $T: \mathbb{R}^{n} \rightarrow \mathbb{R}^{n}$ is a rotation, there exists a constant $C_{0}$, independent of $a, b, \mu$, such that

$$
\forall x \in \mathbb{R}^{n} \forall i, j=1, \ldots, n \quad\left|\frac{\partial\left(F_{a, b, \mu, T}\right)_{i}}{\partial x_{j}}(x)\right| \leq C_{0} \frac{a}{b \mu} .
$$

Therefore, if $a^{\prime} \geq b^{\prime}>0,0<\mu^{\prime}<1, T^{\prime}: \mathbb{R}^{n} \rightarrow \mathbb{R}^{n}$ is a rotation and $G=F_{a^{\prime}, b^{\prime}, \mu^{\prime}, T^{\prime}} \circ F_{a, b, \mu, T}$ then

$$
\forall x \in \mathbb{R}^{n} \forall i, j=1, \ldots, n\left|\frac{\partial G_{i}}{\partial x_{j}}(x)\right| \leq C_{1} \frac{a}{b \mu} \frac{a^{\prime}}{b^{\prime} \mu^{\prime}},
$$

where $C_{1}=n C_{0}^{2}$.

The following results will provide important ingredients for the proof of Theorem 3.1.

Proposition 3.5. Let $n \geq 3, \varepsilon>0$ and $P, Q$ be two points in the interior of $I^{n}$ such that $|P-Q|<\varepsilon$. Let $\delta>0$ be such that $U=V_{\delta}(\overline{P Q})$ is compactly included in the interior of $I^{n}$. If $p<n-1$, then, for all $k \in \mathbb{N}$ there exists $F_{k}=F_{k, P, Q} \in \mathbb{W}_{\lambda}^{1, p}\left(I^{n}\right)$ such that $F_{k}(P)=Q, F_{k}$ sends a neighbourhood of $P$ by a rotation of angle $\pi$ onto a neighbourhood of $Q$, is the identity outside $U$ and

$$
\sup _{k}\left\|F_{k}-I d\right\|_{\infty}<\varepsilon, \quad \lim _{k \rightarrow \infty}\left\|F_{k}-I d\right\|_{1, p}=0 .
$$

In addition, the functions $F_{k}$ are $\mathbb{C}^{\infty}$ diffeomorphisms. 
Proof. If $P=Q$ we choose $F_{k}=I d$. Assume otherwise. The properties that we want to prove are invariant relative to the choice of axis. Therefore, we can assume that the origin is the middle point of the line segment $\overline{P Q}$ and that there exists $c>0$ such that $P=(-c, 0, \ldots, 0)$ and $Q=(c, 0, \ldots, 0)$. Consider $c<a<\varepsilon / 2,0<b \leq a$ such that the ellipsoid $\Sigma_{a, b}$ is contained in $U$. Choose $\mu>0$ such that $(1-\mu) a>c$ and therefore $P, Q \in \Sigma_{a, b, \mu}$. We fix $a$ and $\mu$ and so we drop them from the indexes, denoting $F_{b}=F_{a, b, \mu}$. Of course we have $\left\|F_{b}-I d\right\|_{\infty} \leq 2 a<\varepsilon$. We will prove that $\left\|F_{b}-I d\right\|_{1, p}$ converges to 0 when $b$ tends to 0 . Notice that $\left\|F_{b}-I d\right\|_{p}=\left\|F_{b}-I d\right\|_{L^{p}\left(\Sigma_{a, b}\right)} \leq$ $\varepsilon\left(\lambda\left(\Sigma_{a, b}\right)\right)^{1 / p}=\varepsilon\left(w_{n} a b^{n-1}\right)^{1 / p}$. For the partial derivative we have, by Lemma 3.3

$$
\begin{aligned}
\left\|\frac{\partial\left(F_{b}-I d\right)_{i}}{\partial x_{j}}\right\|_{L^{p}\left(I^{n}\right)} & \leq\left\|\frac{\partial\left(F_{b}\right)_{i}}{\partial x_{j}}\right\|_{L^{p}\left(\Sigma_{a, b}\right)}+\|1\|_{L^{p}\left(\Sigma_{a, b}\right)} \\
& \leq \frac{a}{b} \frac{6 \pi}{\mu} \lambda\left(\Sigma_{a, b}\right)^{1 / p}+\lambda\left(\Sigma_{a, b}\right)^{1 / p} \\
& \leq \frac{6 \pi a}{\mu b} \cdot\left(w_{n} a b^{n-1}\right)^{1 / p}+\left(w_{n} a b^{n-1}\right)^{1 / p} .
\end{aligned}
$$

Then

$$
\left\|F_{b}-I d\right\|_{1, p} \leq \varepsilon\left(w_{n} a b^{n-1}\right)^{1 / p}+n^{2}\left(\frac{6 \pi a}{\mu b} \cdot\left(w_{n} a b^{n-1}\right)^{1 / p}+\left(w_{n} a b^{n-1}\right)^{1 / p}\right)
$$

and the conclusion follows since $\frac{n-1}{p}>1$.

One can prove that, if $p \geq n-1$ then the construction in previous proposition does not work. Of course we only need to consider $p=n-1$. In this case if (for example)

$$
U=\left\{x \in \mathbb{R}^{n}: 1-\frac{\mu}{2} \leq \sqrt{\left(\frac{x_{1}}{a}\right)^{2}+\sum_{i=2}^{n}\left(\frac{x_{i}}{b}\right)^{2}} \leq 1-\frac{\mu}{4}, x_{1}, \ldots, x_{n} \geq 0\right\}
$$

then there exists $C$, independent of $a, b, \mu$ such that $\left\|\frac{\partial(F-I d)_{1}}{\partial x_{n}}\right\|_{L^{p}(U)} \geq C \frac{a^{n}}{\mu^{n-2}}$. The key ingredients when we evaluate the integral are the monotonicity of the derivative of $h_{\mu}$ in $] 1-\frac{\mu}{2}, 1\left[\right.$ and the fact that $h_{\mu}\left(1-\frac{\mu}{4}\right)$ and $h_{\mu}\left(1-\frac{\mu}{2}\right)$ do not depend on $\mu$.

Next result is an upgrade of Proposition 3.5 towards the proof of Theorem 3.1. 
Proposition 3.6. Let $n \geq 3, \varepsilon>0, P, Q, R$ be distinct interior points of $I^{n}$ with $|P-Q|,|P-R|,|Q-R|<\varepsilon$. Consider $\delta>0$ such that $U=V_{\delta}(\overline{P R} \cup \overline{R Q})$ is compactly included in the interior of $I^{n}$ and $\operatorname{diam}(U)<\varepsilon$. Let $p<n-1$.

The function $H_{k}=F_{k, R, Q} \circ F_{k, P, R}$, where $F_{k, R, Q}, F_{k, P, R}$ are given by Proposition 3.5 satisfies $H_{k}(P)=Q$, sends a neighbourhood of $P$ by translation onto a neighbourhood of $Q$, is equal to the identity in $\mathbb{R}^{n} \backslash U$, and

$$
\sup _{k}\left\|H_{k}-I d\right\|_{\infty}<\varepsilon, \quad \lim _{k \rightarrow \infty}\left\|H_{k}-I d\right\|_{1, p}=0 .
$$

Proof. Let $c=\frac{|P-R|}{2}, c^{\prime}=\frac{|Q-R|}{2}$ and $a, a^{\prime}$ be such that $c<a<c+$ $\delta, c^{\prime}<a^{\prime}<c^{\prime}+\delta, a, a^{\prime}<\varepsilon / 2$. Consider $T, T^{\prime}: \mathbb{R}^{n} \rightarrow \mathbb{R}^{n}$ rotations such that $T(-c, 0, \ldots, 0)=P, T(c, 0, \ldots, 0)=R, T^{\prime}\left(-c^{\prime}, 0, \ldots, 0\right)=R$, $T^{\prime}\left(c^{\prime}, 0, \ldots, 0\right)=Q$.

Consider $0<\mu, \mu^{\prime}<1$ such that $(1-\mu) a>c$ and $\left(1-\mu^{\prime}\right) a^{\prime}>c^{\prime}$ and, for $0<b, b^{\prime}<\delta$, the ellipsoids $E_{a, b}=T\left(\Sigma_{a, b}\right)$ and $E_{a^{\prime}, b^{\prime}}=T^{\prime}\left(\Sigma_{a^{\prime}, b^{\prime}}\right)$, which are contained in $U$.

Then, since $a, a^{\prime}, \mu, \mu^{\prime}, T, T^{\prime}$ are fixed we will drop them from the indexes. If $F_{b}=F_{a, b, \mu, T}$ and $G_{b^{\prime}}=F_{a^{\prime}, b^{\prime}, \mu^{\prime}, T^{\prime}}$ and $H_{b, b^{\prime}}=G_{b^{\prime}} \circ F_{b}$, we have $\left\|H_{b, b^{\prime}}-I d\right\|_{\infty}<\operatorname{diam}(U)<\varepsilon$ and

$$
\left\|H_{b, b^{\prime}}-I d\right\|_{L^{p}\left(I^{n}\right)} \leq\|2 \varepsilon\|_{L^{p}\left(E_{a, b} \cup E_{a^{\prime}, b^{\prime}}\right)}=2 \varepsilon \lambda\left(E_{a, b} \cup E_{a^{\prime}, b^{\prime}}\right)^{1 / p} .
$$

Hence, using Corollary 3.4 and noticing that $H_{b, b^{\prime}}=G_{b^{\prime}}$ outside $E_{a, b}$, we obtain

$$
\begin{aligned}
& \left\|\frac{\partial\left(H_{b, b^{\prime}}-I d\right)_{i}}{\partial x_{j}}\right\|_{L^{p}\left(I^{n}\right)} \leq\left\|\frac{\partial\left(H_{b, b^{\prime}}\right)_{i}}{\partial x_{j}}\right\|_{L^{p}\left(E_{a, b} \cup E_{a^{\prime}, b^{\prime}}\right)}+\|1\|_{L^{p}\left(E_{a, b} \cup E_{a^{\prime}, b^{\prime}}\right)} \\
& \leq\left\|\frac{\partial\left(H_{b, b^{\prime}}\right)_{i}}{\partial x_{j}}\right\|_{L^{p}\left(E_{a, b}\right)}+\left\|\frac{\partial\left(G_{b^{\prime}}\right)_{i}}{\partial x_{j}}\right\|_{L^{p}\left(E_{a^{\prime}, b^{\prime}} \backslash E_{a, b}\right)}+\|1\|_{L^{p}\left(E_{a, b} \cup E_{a^{\prime}, b^{\prime}}\right)} \\
& \leq C_{1} \frac{a}{b \mu} \frac{a^{\prime}}{b^{\prime} \mu^{\prime}} \lambda\left(E_{a, b}\right)^{1 / p}+C_{0} \frac{a^{\prime}}{b^{\prime} \mu^{\prime}} \lambda\left(E_{a^{\prime}, b^{\prime}}\right)^{1 / p}+\lambda\left(E_{a, b} \cup E_{a^{\prime}, b^{\prime}}\right)^{1 / p} \\
& \leq \frac{C_{1} a a^{\prime}}{b b^{\prime} \mu \mu^{\prime}}\left(w_{n} a b^{n-1}\right)^{1 / p}+\frac{C_{0} a^{\prime}}{b^{\prime} \mu^{\prime}}\left(w_{n} a^{\prime} b^{\prime n-1}\right)^{1 / p}+\lambda\left(E_{a, b} \cup E_{a^{\prime}, b^{\prime}}\right)^{1 / p} .
\end{aligned}
$$

Choosing $s$ such that $0<s<\frac{n-1}{p}-1$ and $b^{\prime}=b^{s}$ then the conclusion follows letting $b$ converge to 0 . 
Since, by Proposition 3.5, $F_{b}$ restricted to a neighbourhood of $P$ and $G_{b^{\prime}}$ restricted to a neighbourhood of $R$ are rotations of angle $\pi$ on the plane defined by $P, R$ and $Q$, then $H_{b, b^{\prime}}=G_{b^{\prime}} \circ F_{b}$ is locally a translation around $P$.

We can now prove Theorem 3.1.

Proof. (of Theorem 3.1) Let $\mathcal{P}=\left\{P_{i}, Q_{i}: i=1, \ldots, N\right\}$ and $J=\left\{i: P_{i} \neq\right.$ $\left.Q_{i}\right\}$. Let $\left\{R_{i}\right\}_{i \in J}$ be a set of distinct points of the interior of $I^{n}$ not in $\mathcal{P}$ such that $\overline{P_{i} R_{i}} \cap \overline{P_{j} R_{j}}=\overline{R_{i} Q_{i}} \cap \overline{R_{j} Q_{j}}=\emptyset$, if $i \neq j,\left|P_{i}-R_{i}\right|,\left|R_{i}-Q_{i}\right|<\varepsilon / 2$ and

$$
\overline{P_{i} R_{i}} \cap \overline{R_{j} Q_{j}}=\left\{\begin{array}{cl}
R_{i} & \text { if } i=j \\
P_{i} & \text { if } P_{i}=Q_{j} \\
\emptyset & \text { otherwise. }
\end{array}\right.
$$

This can be done by a step by step argument. To avoid overweight of notation, we suppose that $J=I$. First we choose $R_{1}$ in a small ball around the midpoint $\frac{P_{1}+Q_{1}}{2}$ and such that $\left(\overline{P_{1} R_{1}} \cup \overline{R_{1} Q_{1}}\right) \cap \mathcal{P}=\left\{P_{1}, Q_{1}\right\}$. Then, for $i=2, \ldots, N$, we choose $R_{i}$ in a small ball around the midpoint $\frac{P_{i}+Q_{i}}{2}$ and such that $\left(\overline{P_{i} R_{i}} \cup \overline{R_{i} Q_{i}}\right) \cap\left(\mathcal{P} \cup \bigcup_{j=1}^{i-1} \overline{P_{j} R_{j}} \cup \overline{R_{j} Q_{j}}\right)=\left\{P_{i}, Q_{i}\right\}$.

Let $\delta>0$ be such that, if $L_{1}, L_{2}$ are two line segments, each one of the form $\overline{P_{i} R_{i}}$ or $\overline{R_{j} Q_{j}}$, then $V_{\delta}\left(L_{1}\right) \cap V_{\delta}\left(L_{2}\right)=\emptyset$ if and only if $L_{1} \cap L_{2}=\emptyset$ (see Figure 1).

For each $i \in I$, consider ellipsoids $E_{i}$ and $\tilde{E}_{i}$ such that $\overline{P_{i} R_{i}} \subseteq E_{i} \subseteq$ $V_{\delta}\left(\overline{P_{i} R_{i}}\right)$ and $\overline{R_{i} Q_{i}} \subseteq \tilde{E}_{i} \subseteq V_{\delta}\left(\overline{R_{i} Q_{i}}\right)$.

Let $G_{k, i}=F_{k, P_{i}, R_{i}}$ and $H_{k, i}=F_{k, R_{i}, Q_{i}}$ be given by Proposition 3.5, if $i \in I$, and $G_{i}=H_{i}=I d$, otherwise.

Define $G_{k}=G_{k, 1} \circ \cdots \circ G_{k, N}, H_{k}=H_{k, 1} \circ \cdots \circ H_{k, N}$ and $F_{k}=H_{k} \circ G_{k}$. We have that,

$$
\begin{aligned}
F_{k}-I d=\sum_{i, j} \chi_{E_{i} \cap G_{k, i}^{-1}\left(\tilde{E}_{j}\right)}\left(H_{k, j} \circ G_{k, i}-I d\right)+ & \sum_{i} \chi_{E_{i} \backslash\left(\cup_{j} G_{k, i}^{-1}\left(\tilde{E}_{j}\right)\right)}\left(G_{k, i}-I d\right) \\
& +\sum_{j} \chi_{\tilde{E}_{j} \backslash\left(\cup_{i} E_{i}\right)}\left(H_{k, j}-I d\right) .
\end{aligned}
$$

Notice that, when applying the function $F_{k}-I d$ to a point, at most one of the terms is non-zero. Then, by the choice of the points $R_{i}$, we have that

$$
\left\|F_{k}-I d\right\|_{\infty}=\max _{i, j}\left\{\left\|H_{k, j} \circ G_{k, i}-I d\right\|_{\infty},\left\|G_{k, i}-I d\right\|_{\infty},\left\|H_{k, j}-I d\right\|_{\infty}\right\}<\varepsilon .
$$




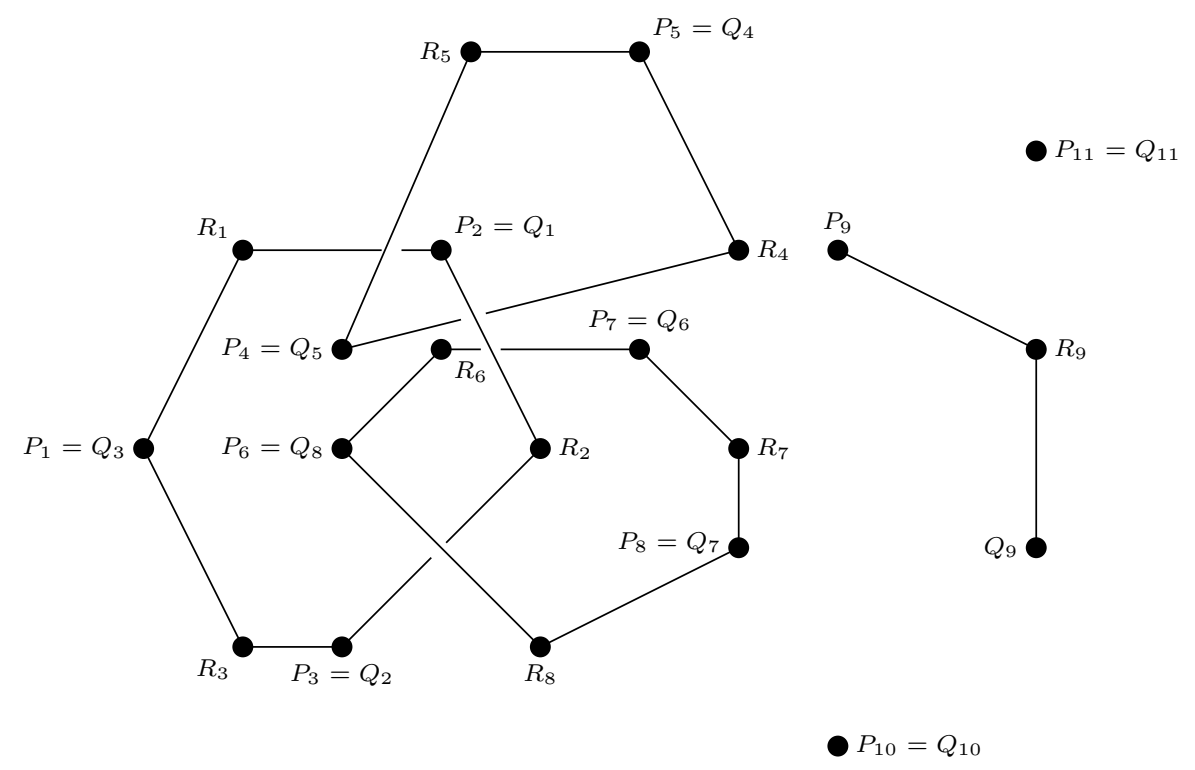

Figure 1: An illustrative example of the setting of Theorem 3.1.

Furthermore,

$$
\begin{aligned}
\left\|F_{k}-I d\right\|_{1, p} & \leq \sum_{i, j}\left\|H_{k, j} \circ G_{k, i}-I d\right\|_{1, p} \\
& +\sum_{i}\left\|G_{k, i}-I d\right\|_{1, p}+\sum_{j}\left\|H_{k, j}-I d\right\|_{1, p} .
\end{aligned}
$$

Hence, the conclusion follows from the previous proposition.

\section{Volume preserving Sobolev weak Lusin theorem}

In this section we prove a volume preserving weak Lusin theorem for the Sobolev class $\mathbb{W}_{\lambda}^{1, p}(X)$, for $p<n-1$ and show that if $p>n$ the result is not true. 
Theorem A (Volume preserving Sobolev weak Lusin theorem). Let $X$ be a closed connected n-dimensional manifold, $n \geq 3$. Let $\varepsilon>0$ and $g \in \mathcal{G}(X)$ with $\|g-I d\|_{\infty}<\varepsilon$.

Let $1 \leq p<n-1$. Then given any weak topology neighbourhood $\mathcal{W}$ of $g$, there exists $f \in \mathbb{W}_{\lambda}^{1, p}(X)$ such that $f \in \mathcal{W}$ and $\|f-I d\|_{\infty ; 1, p}<\varepsilon$.

In addition, $f \in \mathbb{W}_{\lambda}^{1, \infty}(X)$.

Since any smooth closed connected $n$-manifold can be obtained from $I^{n}$ by making boundary identifications, it follows, using a Moser's result [8], that the proof of Theorem A reduces to the proof on the unit cube. For this, we will follow the strategy of the proof of [3, Theorem 6.2], adapting it to the Sobolev setting. As a key step, we will prove that a dyadic permutation of $I^{n}$ can be approximated by a Sobolev- $(1, p)$ volume preserving homeomorphism of $I^{n}$, in the sense described in Theorem 4.1.

We recall that a dyadic permutation of $I^{n}$ of order $m$ is a bijection $\mathscr{P}: I^{n} \rightarrow I^{n}$ which permutes by simple translation the dyadic open cubes that are products of intervals of the form $] k / 2^{m},(k+1) / 2^{m}[$.

Theorem 4.1. Let $\varepsilon>0$ and $\mathscr{P}$ be a dyadic permutation of the cube $I^{n}$, $n \geq 3$, with $\|\mathscr{P}-I d\|_{\infty}<\varepsilon$.

Let $1 \leq p<n-1$. Then given any $\gamma>0$, there is $f \in \mathbb{W}_{\lambda}^{1, p}\left(I^{n}\right)$, with $\|f-I d\|_{\infty ; 1, p}<\varepsilon$, and equal to the identity on a neighbourhood of the boundary, satisfying

$$
\lambda\{x: \mathscr{P}(x) \neq f(x)\}<\gamma .
$$

In addition, $f \in \mathbb{W}_{\lambda}^{1, \infty}\left(I^{n}\right)$.

Proof. Without loss of generality we can suppose that $\mathscr{P}$ is a permutation of dyadic cubes $\sigma_{i}, i=1, \ldots, N$, with diameter less than $\left(\varepsilon-\|\mathscr{P}-I d\|_{\infty}\right) / 3$. For $0<\beta<1$, we denote by $\sigma_{i}^{\beta}$ the cubes concentric to $\sigma_{i}$ with parallel faces and such that $\lambda\left(\sigma_{i}^{\beta}\right)=\beta \lambda\left(\sigma_{i}\right)$. We denote by $P_{i}$ the center of the cube $\sigma_{i}$ and let $Q_{i}=\mathscr{P}\left(P_{i}\right), i=1, \ldots, N$. By hypothesis, $\left|P_{i}-Q_{i}\right|<\varepsilon$ for every $i$. Since $\|\mathscr{P}-I d\|_{\infty}<\frac{1}{3} \varepsilon+\frac{2}{3}\|\mathscr{P}-I d\|_{\infty}$, applying Theorem 3.1 to the sets $\left\{P_{i}\right\}_{i=1}^{N}$ and $\left\{Q_{i}\right\}_{i=1}^{N}$, we obtain a volume preserving $C^{\infty}$ diffeomorphism of $I^{n}$ equal to the identity on a neighbourhood of the boundary of $I^{n}$ and

$$
\|F-I d\|_{\infty ; 1, p}<\frac{1}{3} \varepsilon+\frac{2}{3}\|\mathscr{P}-I d\|_{\infty} .
$$


Furthermore, since $F$ sends a neighbourhood of $P_{i}$ by translation onto a neighbourhood of $Q_{i}$, there exists $0<\alpha<1$ such that $F=\mathscr{P}$ on $\sigma_{i}^{\alpha}$, for every $i$. Consequently, $\lambda\{x: \mathscr{P}(x) \neq F(x)\} \leq 1-\alpha$. If $1-\alpha<\gamma$, the Theorem is proved taking $f=F$. Otherwise, it is clearly enough to obtain a map $f$ which coincides with $\mathscr{P}$ on the cubes $\sigma_{i}^{\beta}$, for some $\beta$ such that $\beta>1-\gamma$. Fix $\beta>1-\gamma$. To obtain $f$ we first define a map $T: I^{n} \rightarrow I^{n}$ (not volume preserving) which leaves each cube $\sigma_{i}$ invariant in the following way: $T$ sends $\sigma_{i}^{\alpha}$ radially onto $\sigma_{i}^{\beta}$, with constant Jacobian matrix $J T=\sqrt[n]{\frac{\beta}{\alpha}} \cdot I d$, and sends $\sigma_{i} \backslash \sigma_{i}^{\alpha}$ radially onto $\sigma_{i} \backslash \sigma_{i}^{\beta}$, with constant Jacobian matrix $J T=$ $\sqrt[n]{\frac{1-\beta}{1-\alpha}} \cdot I d$.

Finally, we define the map $f:=T F T^{-1}$. This map satisfies the following:

(i) $\lambda\{x: \mathscr{P}(x) \neq f(x)\}<\gamma$, by construction.

(ii) $J f(x)=J F\left(T^{-1}(x)\right)$, except in the boundary of $\sigma_{i}, \sigma_{i}^{\alpha}$ and $\sigma_{i}^{\beta}$. For this, just notice that $x \in \cup_{i} \operatorname{int}\left(\sigma_{i}^{\beta}\right)$ if and only if $\left.f\left(T^{-1}\right)(x)\right) \in \cup_{i} \operatorname{int}\left(\sigma_{i}^{\alpha}\right)$.

(iii) $f$ is volume preserving. Since, by (ii), $J f(x)=J F\left(T^{-1}(x)\right)$, except in the boundary of $\sigma_{i}, \sigma_{i}^{\alpha}$ and $\sigma_{i}^{\beta}$, we have that $\operatorname{det} J f(x)=1$.

We will now obtain the control of $\|f-I d\|_{\infty ; 1, p}$. By Theorem 3.1, we have that $\|f-I d\|_{\infty} \leq\|F-I d\|_{\infty}+2\|T-I d\|_{\infty}<\varepsilon$.

Furthermore, using (ii), the change of variables defined by $T^{-1}$ and the fact that $F=\mathscr{P}$ in $\sigma_{i}^{\alpha}$, we obtain that

$$
\begin{aligned}
\sum_{j, k}\left\|\frac{\partial(f-I d)_{k}}{\partial x_{j}}\right\|_{p}^{p} & =\sum_{j, k} \int_{I^{n}}\left|\frac{\partial(F-I d)_{k}}{\partial x_{j}}\left(T^{-1}(x)\right)\right|^{p} d x \\
& =\sum_{j, k} \int_{I^{n}}\left|\frac{\partial(F-I d)_{k}}{\partial x_{j}}(x)\right|^{p} \operatorname{det} J T(x) d x \\
& =\sum_{j, k} \int_{I^{n} \backslash \cup \sigma_{i}^{\alpha}}\left|\frac{\partial(F-I d)_{k}}{\partial x_{j}}(x)\right|^{p} \operatorname{det} J T(x) d x \\
& =\frac{1-\beta}{1-\alpha} \sum_{j, k} \int_{I^{n} \backslash \cup \sigma_{i}^{\alpha}}\left|\frac{\partial(F-I d)_{k}}{\partial x_{j}}(x)\right|^{p} d x \\
& \leq \frac{1-\beta}{\gamma} \sum_{j, k}\left\|\frac{\partial(F-I d)_{i}}{\partial x_{j}}\right\|_{p}^{p} .
\end{aligned}
$$

Hence, taking $\beta$ large enough, the conclusion follows. 
We can now complete the proof of Theorem A.

Proof. (of Theorem A) Let $\varepsilon>0$ and $g \in \mathcal{G}\left(I^{n}\right)$ with $\|g-I d\|_{\infty}<\varepsilon$. Let $\delta, \gamma>0$. We will obtain $f \in \mathbb{W}_{\lambda}^{1, p}\left(I^{n}\right)$, with $\|f-I d\|_{\infty ; 1, p}<\varepsilon$ and equal to the identity on the boundary of $I^{n}$, satisfying $\lambda\{x:|g(x)-f(x)| \geq \delta\}<\gamma$.

The automorphism $g \in \mathcal{G}\left(I^{n}\right)$ can be weakly approximated by a dyadic permutation $R$ with $\rho(g, R)$ small. This follows from the denseness of dyadic permutations in $\mathcal{G}\left(I^{n}\right)$, in the weak topology (see [2, §2] or [3, Lemma 6.4]).

Furthermore, the dyadic permutation $R$ can be weakly approximated by another dyadic permutation $\mathscr{P}$ such that $\lambda\{x:|g(x)-\mathscr{P}(x)| \geq \delta\}<\gamma$ and $\|\mathscr{P}-I d\|_{\infty}<\varepsilon$. The technique for this approximation is described in the proof of $[3$, Theorem 6.2, p. 46].

Set $\gamma_{0}:=\gamma-\lambda\{x:|g(x)-\mathscr{P}(x)| \geq \delta\}$. Applying Theorem 4.1 to the permutation $\mathscr{P}$ we obtain a map $f \in \mathbb{W}_{\lambda}^{1, p}\left(I^{n}\right)$, with $\|f-I d\|_{\infty ; 1, p}<\varepsilon$, and equal to the identity on a neighbourhood of the boundary, satisfying $\lambda\{x: \mathscr{P}(x) \neq f(x)\}<\gamma_{0}$. Hence, $\lambda\{x:|g(x)-f(x)| \geq \delta\}<\gamma$. In addition, $f \in \mathbb{W}_{\lambda}^{1, \infty}\left(I^{n}\right)$.

Example 4.1 (Counterexample to Theorem A for $p>n$ ). Consider $p>$ $n$ and $g$ any continuous element of $\mathcal{G}\left(I^{n}\right)$ different from the identity. Let $\varepsilon_{0}>0$ and $x_{0} \in I^{n}$ be such that

$$
\left|g\left(x_{0}\right)-x_{0}\right|=\|g-I d\|_{\infty}=\varepsilon_{0} .
$$

For $k>0$ let $D_{k}=\left\{x \in I^{n}:\left|x-x_{0}\right|<\frac{1}{k},\left|g(x)-g\left(x_{0}\right)\right|<\frac{1}{k}\right\}$. Notice that $\delta_{k}=\lambda\left(D_{k}\right)>0$, since $D_{k}$ is a non-empty open set.

If the weak Lusin Theorem was valid for $\mathbb{W}_{\lambda}^{1, p}\left(I^{n}\right)$ then, for $k \in \mathbb{N}$ there would exist $f_{k} \in \mathbb{W}_{\lambda}^{1, p}\left(I^{n}\right)$ such that

$\left\|f_{k}-I d\right\|_{\infty ; 1, p}<\varepsilon_{0}+\frac{1}{k}, \lambda\left(A_{k}\right)<\delta_{k}$, where $A_{k}=\left\{x \in I^{n}:\left|f_{k}(x)-g(x)\right| \geq \delta_{k}\right\}$.

By construction $D_{k} \nsubseteq A_{k}$. Consider $x_{1} \in D_{k} \backslash A_{k}$. Then

$$
\left\|f_{k}-I d\right\|_{\infty} \geq\left|f_{k}\left(x_{1}\right)-x_{1}\right| \geq\left|g\left(x_{1}\right)-x_{1}\right|-\left|g\left(x_{1}\right)-f_{k}\left(x_{1}\right)\right| \geq \varepsilon_{0}-\frac{2}{k}-\delta_{k} .
$$

Since $\left\|f_{k}-I d\right\|_{\infty ; 1, p}=\left\|f_{k}-I d\right\|_{\infty}+\left\|D\left(f_{k}-I d\right)\right\|_{p}$, we obtain $\| D\left(f_{k}-\right.$ $I d) \|_{p} \leq \frac{3}{k}+\delta_{k}$. As $f_{k}=I d$ on the boundary of $I^{n}$ we conclude that the sequence $\left(f_{k}\right)_{k \in \mathbb{N}}$ converges to $I d$ in $W^{1, p}\left(I^{n}\right)$. Moreover, as $p>n$ then 
$\left(f_{k}\right)_{k \in \mathbb{N}}$ converges to Id in $C^{0}\left(I^{n}\right)$ which is absurd because $\left\|f_{k}-I d\right\|_{\infty} \geq$ $\varepsilon_{0}-\frac{2}{k}-\delta_{k}$.

We observe that, this example also shows that Theorem $A$ is not valid for $\mathbb{C}_{\lambda}^{0, \alpha}\left(I^{n}\right)$, for all $0<\alpha \leq 1$. Indeed, as before, we obtain $f_{k} \in \mathbb{C}_{\lambda}^{0, \alpha}\left(I^{n}\right)$ such that

$$
\left\|f_{k}-I d\right\|_{\infty} \geq \varepsilon_{0}-\frac{2}{k}-\delta_{k}, \quad \sup _{x \neq y} \frac{\left|\left(f_{k}-I d\right)(x)-\left(f_{k}-I d\right)(y)\right|}{|x-y|^{\alpha}}<\frac{3}{k}+\delta_{k} .
$$

Choosing y such that $f_{k}(y)=y$ we have $\left|\left(f_{k}-I d\right)(x)\right|<\left(\frac{3}{k}+\delta_{k}\right)\left(\operatorname{diam}\left(I^{n}\right)\right)^{\alpha}$, which is a contradiction.

\section{Acknowledgements}

The authors would like to thank the anonymous referee for the careful reading of the manuscript and for giving very helpful comments and suggestions.

AA and MJT were partially supported by the Research Centre of Mathematics of the University of Minho with the Portuguese Funds from the "Fundação para a Ciência e a Tecnologia", through the Project UID/MAT/ $00013 / 2013$.

MB was partially supported by FCT - 'Fundação para a Ciência e a Tecnologia', through Centro de Matemática e Aplicações (CMA-UBI), Universidade da Beira Interior, project UID/MAT/00212/2013.

\section{References}

[1] Steve Alpern, Approximation to and by measure preserving homeomorphisms, J. London Math. Soc., 18(2) (1978), 305-315.

[2] Steve Alpern and Robert D. Edwards, Lusin's theorem for measure preserving homeomorphisms, Mathematika, 26(1) (1979), 33-43.

[3] Steve Alpern and Vidhu S. Prasad, Typical Dynamics of Volume Preserving Homeomorphisms, Cambridge Tracts in Mathematics, 2000.

[4] Edson de Faria, Peter Hazard, Charles Tresser, Infinite entropy is generic in Hölder and Sobolev spaces, C. R. Acad. Sci. Paris, Ser. I, 355(11) (2017), 1185-1189. 
[5] Edson de Faria, Peter Hazard, Charles Tresser, Genericity of Infinite Entropy for Maps with Low Regularity, Preprint ArXiv 2017.

[6] Paul R. Halmos, Lectures on ergodic theory, The Mathematical Society of Japan, 1956. Publications of the Mathematical Society of Japan, no. 3.

[7] Tadeusz Iwaniec, Leonid V. Kovalev and Jani Onnine, Diffeomorphic approximation of Sobolev homeomorphisms. Arch. Rational Mech. Anal., 201 (2011), 1047-1067.

[8] Jürgen Moser, On the volume elements of a manifold, Trans. Amer. Math. Soc., 120 (1965), 286-294.

[9] John C. Oxtoby and Stanislaw M. Ulam, Measure-preserving homeomorphisms and metrical transitivity, Ann. of Math., 42(4) (1941), 874-920.

[10] John C. Oxtoby, Approximation by measure-preserving homeomorphisms, Recent Advances in Topological Dynamics, Proc. Conf. in Topological Dynamics, Yale University, New Haven, Conn., 1972; in honor of Gustav Arnold Hedlund, (Lecture Notes in Mathematics, 318) Springer, Berlin, 1973, 206-217.

[11] John C. Oxtoby, Measure and category. A survey of the analogies between topological and measure spaces, volume 2 of Graduate Texts in Mathematics, Springer-Verlag, New York, second edition, 1980.

[12] H. E. White Jr., The approximation of one-one measurable transformations by measure preserving homeomorphisms, Proc. Amer. Math. Soc., 44 (1974), 391-394. 